\title{
Extradural Dermoid Cyst of the Anterior Infratemporal Fossa. Case Report
}

\author{
Kentaro Watanabe ${ }^{1}$ Carol A. Filomena ${ }^{2}$ Yoichi Nonaka ${ }^{1} \quad$ Masahide Matsuda $^{3} \quad$ Ali R. Zomorodi ${ }^{1}$ \\ Allan H. Friedman ${ }^{1}$ Takanori Fukushima ${ }^{1}$
${ }^{1}$ Division of Neurosurgery, Duke University Medical center, Durham, North Carolina, United States North Carolina, United States
${ }^{3}$ Department of Neurosurgery, Faculty of Medicine, University of \\ 2 Department of Pathology, Duke University Medical center, Durham, \\ Tsukuba, Tsukuba, Ibaraki, Japan \\ J Neurol Surg Rep 2015;76:e195-e199.

\begin{abstract}
Address for correspondence Kentaro Watanabe, MD, Division of Neurosurgery, Duke University Medical Center, Box3807, 1000 Trent Drive, 4520 Hospital South, Durham, NC 27710, United States (e-mail: kentarow31@gmail.com).
\end{abstract}

\author{
Abstract \\ Keywords \\ - dermoid cyst \\ - anterior \\ infratemporal fossa \\ - extradural
}

\begin{abstract}
Dermoid cysts are rare in the skull base. There have been 10 reported cases of dermoid cysts in the cavernous sinus, two in the petrous apex, and one in the extradural Meckel cave. This is the first case report of a dermoid cyst in the anterior infratemporal fossa attached to the anterior dura of the foramen ovale. The clinical presentation, radiologic findings, histologic features, tumor origin, and operative technique are described along with a review of the literature.
\end{abstract}

\section{Introduction}

Dermoid cysts are benign congenital tumors that occur in the extradural and intradural cranial spaces. Most intradural cases occur in the midline area involving the suprasellar, parasellar, frontobasal, and spinal cord as well as the posterior fossa. ${ }^{1-8}$ Extradural dermoid cysts may occur in the orbit, fontanel, or any bone of the calvarium. ${ }^{8-11}$ Extradural dermoid cysts in the skull base, however, are rare. We report the case of a 55-year-old woman with a dermoid cyst of the extradural infratemporal fossa. The tumor was attached to the dura of the foramen ovale without touching the cavernous sinus lateral wall, and it expanded and eroded the anterior infratemporal bone and pterygoid process. Intraoperative findings and histologic examination showed that the tumor was a typical dermoid cyst.

\section{Case Report}

A 55-year-old woman presented with narcoleptic symptoms, slurred speech, and depression. Computed tomography (CT) and magnetic resonance image (MRI) examination revealed a large bone defect or dehiscence at the anterior infratemporal fossa ( - Fig. 1A). The lesion had a heterogeneous hyperintense signal on T1-weighted (-Fig. 1B, C), T2-weighted, and fluidattenuated inversion recovery images. The margin was well demarcated with no appreciable contrast enhancement. Because the nature of the expanding mass lesion compressing the anterior basal temporal lobe needed to be determined, microsurgical resection for histologic confirmation was indicated.

\section{Operation}

The patient was placed in the supine position with her head rotated to the left and supported with a three-pin head clamp. A standard preauricular temporal incision was made. The scalp was elevated with the entire temporal muscle and periosteum in a single layer. A standard subtemporal craniotomy was performed, and extradural dissection was advanced to the foramen ovale and rotundum. The anterior subtemporal bone was removed, skeletonizing the infratemporal fascia, unroofing the foramen rotundum, and exposing the mass. The foramen ovale was enlarged, and the posterior margin of the tumor mass was adhered to the dura of the mandibular nerve ( - Fig. 2A). The tumor was an encapsulated mass containing typical soft yellowish received

February 10, 2014

accepted after revision

November 21, 2014

published online

October 29, 2015
DOI http://dx.doi.org/

10.1055/s-0034-1544111. ISSN 2193-6358. (c) 2015 Georg Thieme Verlag KG
Stuttgart · New York

License terms

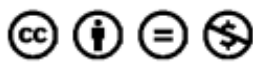



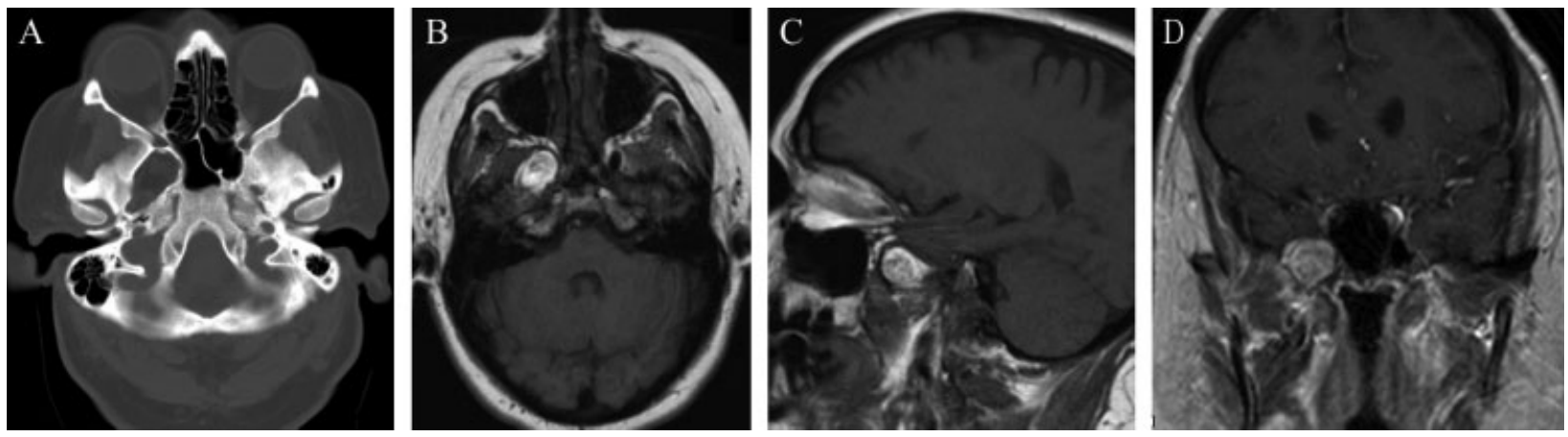

Fig. 1 (A) Computed tomography bone window image. Bone defect in the right anterior inferior fossa. (B) Magnetic resonance imaging (MRI) T1-weighted axial image. Heterogeneous hyperintensity lesion in the right anterior inferior fossa. (C) MRI T1-weighted sagittal image. Heterogeneous hyperintensity lesion in the right anterior inferior fossa. (D) MRI T1-weighted coronal image. Heterogeneous hyperintensity lesion in the right foramen ovale.

butter-like semisolid material and hair, indicating a dermoid cyst. The tumor capsule and the surrounding tissue were connected with the pterygoid venous system. The tumor capsule was elevated from the parapharyngeal wall and the pterygoid process and separated from the posterior wall of the maxillary sinus. The tumor including its fibrous

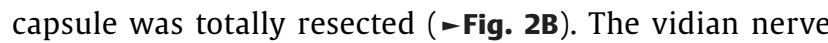
was not seen in this operation. Most of the fibers of the third branch of the trigeminal nerve were preserved. After total resection of the tumor, hemostasis was secured, particularly around the infratemporal pterygoid venous plexus. Cranioplasty was performed with titanium mesh to reconstruct the skull defect. The postoperative course was uneventful, and the patient was discharged. There was no recurrence during 6 months of follow-up.

\section{Pathology}

Histologic examination confirmed the diagnosis of a benign dermoid cyst. The tumor consisted of a benign cyst with an irregularly thickened thick fibrous wall with focal ossification and mature bone formation ( - Fig. 3A, 3C). A definite epithelial lining was not present, but focal cutaneous adnexal structures ( - Fig. 3B) and hair ( - Fig. 3D) were identified. Evidence of malignant transformation, rarely reported to occur, was not present.

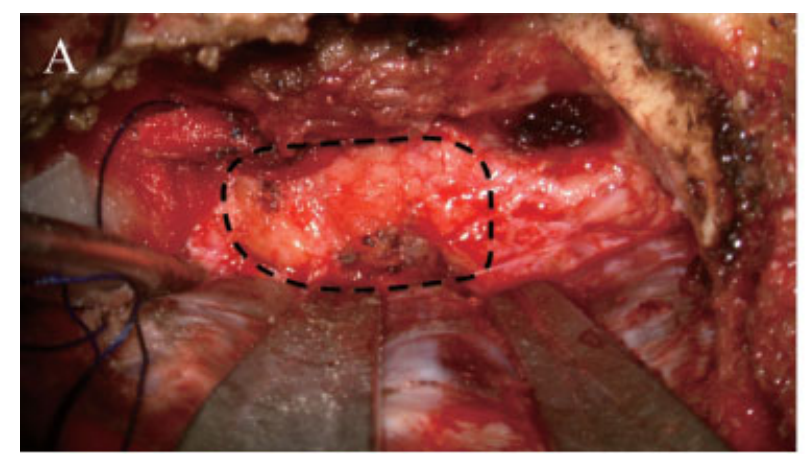

\section{Discussion}

\section{Dermoid Characteristics}

Dermoid cysts are presumed to arise from included fragments of the primitive ectodermal layer and mesenchymal elements within the closing neuroectodermal folds during weeks 3 to 5 of fetal life. Most dermoid cysts are reported during childhood and have a predilection to occur in the midline. ${ }^{1,7,10}$ They may occur in intradural or extradural spaces and may occur anywhere within the cranial vault. Intradural lesions are more common in the posterior fossa around the cerebellar vermis and the fourth ventricle, in the spinal canal, and, less frequently, in the supraparasellar, pineal, and parapontine regions. ${ }^{7,12,13}$ Extradural lesions have been reported in the fontanels, periorbital, nasal, frontotemporal, eustachian tube, parietal, occipital, cavernous sinus, and petrous apex locations. ${ }^{11,14-17}$ They tend to originate from skull sutures located near the skin and have direct connections between the dermal sinus and intradural cyst. In these extradural dermoid cysts, only three cases were reported as lateral skull base dermoid lesions. ${ }^{8,9,11}$

Patients may be asymptomatic or have only mild symptoms for extended periods of time. A psychoneurotic symptom is often experienced by many patients regardless of cyst location. ${ }^{9,18}$ Infrequently, intracranial dermoid cysts rupture

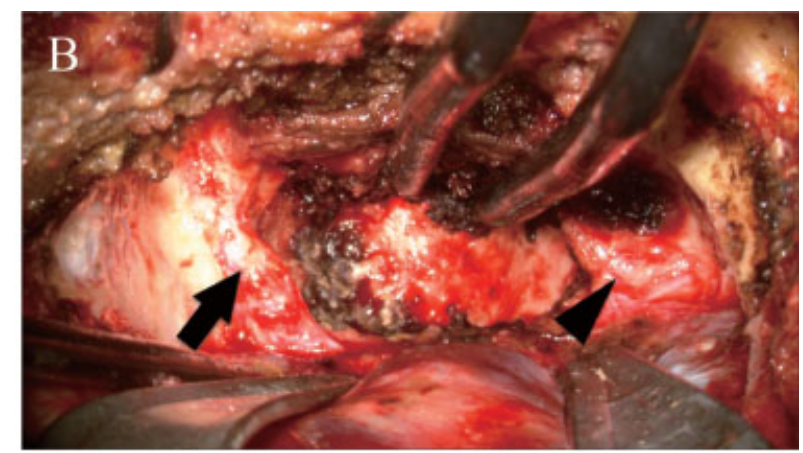

Fig. 2 (A) Intraoperative view after drilling the anterior infra temporal fossa bone and foramen rotundum and foramen ovale were skeletonized. Dashed circle indicates the tumor. (B) Intraoperative view after removing the tumor. The lateral pterygoid plate was exposed between the trigeminal maxillary nerve (arrow) and trigeminal mandibular nerve (arrowhead). 

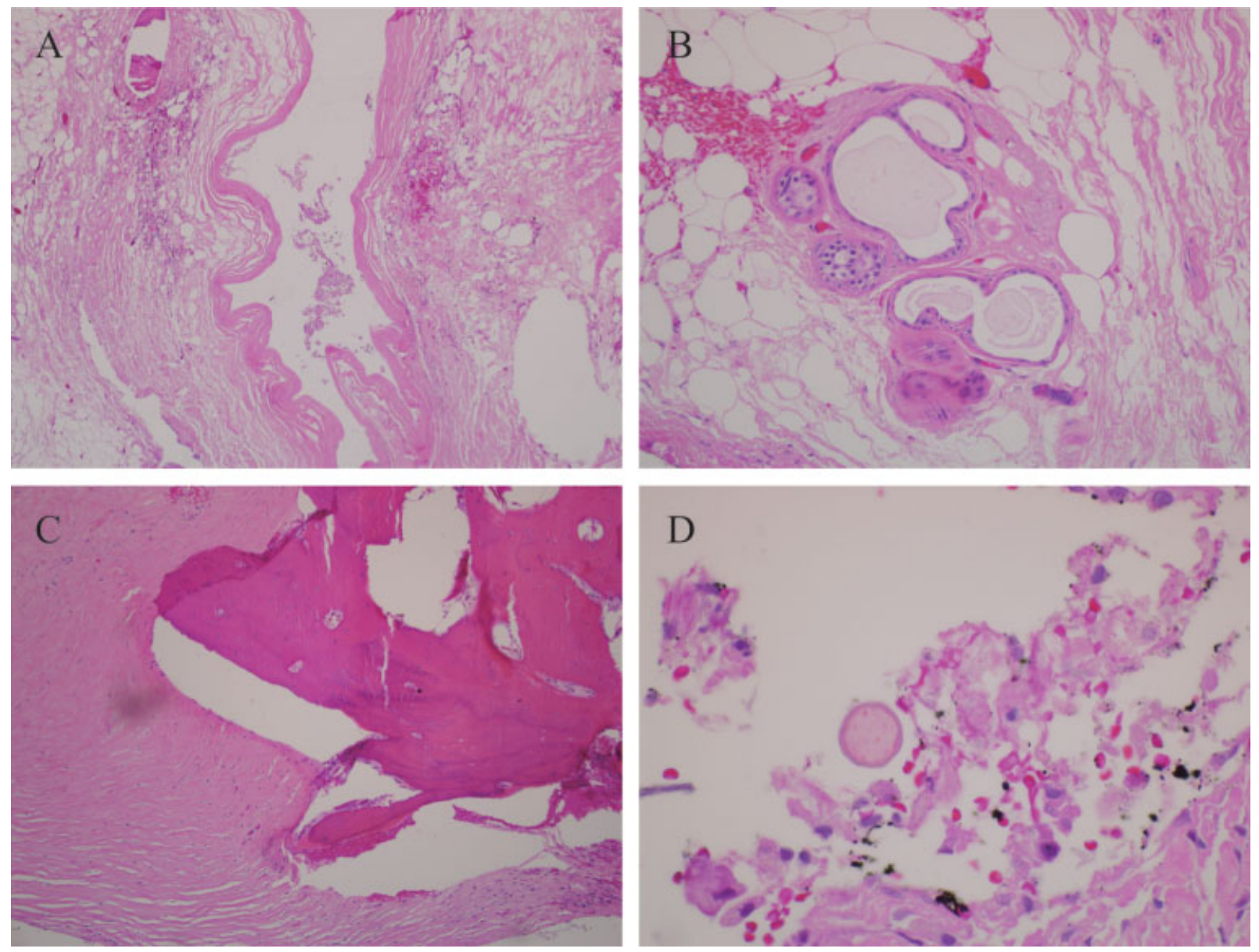

Fig. 3 (A) Simple cyst lining without residual squamous epithelium. (B) Focal cutaneous adnexal structures are identified in the cyst wall that distinguishes the dermoid cyst from an epidermoid cyst. (C) Irregularly thickened fibrous wall with focal ossification with mature bone formation. (D) Focal cross sections of hair are identified.

incidentally or following trauma. ${ }^{6,12,18,19}$ The cyst contents are scattered within the cranial space and elicit a strong inflammatory response that may result in ventriculitis, nonbacterial (chemical) meningitis, chronic granulated arachnoiditis, aqueductal stenosis, or seizure. ${ }^{5,6,18}$ Skull base patients had headache and phantosmia, staring spells, and difficulties with concentration and memory. In the present case, the patient experienced narcoleptic symptoms and depression.

Dermoid cysts are usually lined by stratified squamous epithelium. The cyst wall is irregularly thickened and contains cutaneous adnexal structures, and it may have mature bone formation. In this case, the cyst content consisted of hair and sebaceous debris.

\section{Radiologic Findings}

Dermoid cysts are often confidently diagnosed with CT and MRI. These cysts are typically well-circumscribed masses of dense fat without contrast enhancement. The cyst walls occasionally show peripheral contrast enhancement and focal calcifications. The cysts appear hypodense with negative attenuation values on $\mathrm{CT}^{2,20,21}$ On MRI, fatty contents, calcifications, hair follicles, or epithelial debris give these cysts heterogeneous hypointensity in the solid tumor part on
T1-weighted images and inhomogeneous hyperintensity on T2-weighted images. ${ }^{20}$ Fat-suppressed T1-weighted images help in the differential diagnosis of dermoid cysts from arachnoid cysts, cystic gliomas, or others. For an extradural skull base lesion, the differential diagnosis for dermoid cysts includes epidermoids, chordomas, chondrosarcomas, meningoceles, and metastatic tumors.

The patient's age, clinical history, location of the lesion, presence of calcifications, and heterogeneous or homogeneous values are useful in the preoperative evaluation. In this case, the radiologic findings led to a preoperative diagnosis of lipid tumor including dermoid cyst. CT scan demonstrated a markedly hypodense area in the anterior infratemporal fossa and a widened foramen ovale with osseous erosion. MRI demonstrated heterogeneous high signal intensity on T1-weighted images and hyposignal intensity on T2-weighted images.

\section{Treatment}

Dermoid cysts are benign tumors, although malignant transformation to squamous cell carcinoma has been reported. Surgery is the only effective treatment with the goal of a complete resection of the lesion to prevent recurrence. 
Complete resection may not be feasible due to fibrous adhesion of the tumor capsule with the adjacent neural and vascular structures. In fact, some authors have reported that an aggressive surgical approach has resulted in a high mortality and morbidity rate. ${ }^{4}$ However, partial or subtotal resection may result in tumor recurrence or chemical meningitis from leakage of the cyst contents. In the present case, the extradural anterior infratemporal fossa approach was an effective and less invasive surgical approach. Total resection was accomplished. The pterygoid venous plexus, trigeminal nerve, and vidian nerve were preserved during surgery. Because the dermoid tumor may elicit an inflammatory response, the risk of infection should be anticipated. The patient should be administered antibiotics before, during, and after surgery. Dermoid cysts that are removed intact are unlikely to spill cyst contents into the surgical space with the resulting inflammatory response. During the surgery, irrigation with lactate Ringer's solution minimizes potential complications.

In the present case, the middle fossa approach was simply an approach to the middle fossa lesion. Several otologic reports have used a transvestibular approach or endoscopic transantral approach to the middle fossa lesions. However, the transvestibular approach requires mastoidectomy, and the corridor is narrow and deep. We found that the endoscopic transantral approach is also useful. However, the transcranial anterior middle fossa approach can reach the lesion directly extradurally and control venous plexus bleeding from the pterygoid plexus.

\section{Origin of the Tumor}

Authors of the few reports of extradural dermoid cysts have described tumors in the posterior fossa and in the diploe of the anterior fontanel, orbital regions, frontotemporal, parietal, or occipital locations. The location of the lesion in this report is extremely unusual because it was not located in the midline, posterior fossa, cavernous sinus, or any reported locations.

It is general knowledge that paraxial mesodermal differentiate into dura mater. Ammirati et al reported extradural dermoid tumor of the petrous apex with tumor compression of the gasserian ganglion and the mesial temporal lobe superiorly. ${ }^{9}$ Uppal et al reported a dermoid cyst of the infratemporal fossa; however, the location was in the posterior infra temporal fossa. ${ }^{8}$ Although several theories have been proposed to explain the existence of stereotypical regions for dermoid cysts, the exact etiology remains unknown. Caldarelli et al suggested that dermoid cysts located in lateral or intraparenchymal positions are anomalies that may be explained by the displacement of the primitive ectodermal and mesodermal cells caused by the developing cerebral vasculature along the Virchow-Robin spaces. ${ }^{1}$ Nakagawa et al defined intradural dermoid cyst of the cavernous sinus with a clearly anatomical relationship. ${ }^{22}$ Lunardi and Missori reported cavernous dermoid cysts with the observation that intracranial intradural dermoid cysts appear near or adherent to large venous structures with dermal structures or venous channels derived from the mesoderm. ${ }^{4}$ They proposed the hypothesis that vascular migration is responsible for the development of dermoid cysts through the entrapment of mesodermal nonvascular cells. North et al reported a dermoid case located between the dural layers without invasion into the cavernous sinus. ${ }^{16}$ Fujimaki et al reported that most extradural dermoids cysts are located between the galea and pericranium. ${ }^{15}$ And they found the dermoid cyst located underneath the periosteum and its stalk attached to the dura. This report indicates dermoid cysts can possibly arise from the dura or suture.

In our cases, during removal of the dermoid cyst, the dermoid cyst adhered and bled from the pterygoid plexus; the attachment with the foramen ovale and trigeminal nerve third branch was severe. However the dermoid cysts did not attach to the cavernous sinus wall. The dermoid cyst was separated with bone from the cavernous sinus. These findings suggest three possible hypotheses concerning the origin of the dermoid cyst in this case. It may have originated from (1) ectodermal and mesodermal cells that may have migrated along the venous channels connecting the cavernous sinus with the pterygoid plexus, (2) the intra- or interdural fold of the foramen ovale dura propria, or (3) the sphenotemporal suture.

\section{Conclusion}

A rare case of an extradural anterior infratemporal fossa dermoid cyst was reported. The tumor was totally removed through a preauricular infratemporal fossa approach. Dermoid cysts may arise anywhere in the intra- and extracranial space. Although they are benign tumors that expand slowly, they may cause a marked inflammatory response and destroy surrounding tissue structures. Radical total resection for cure is the best management, and a sophisticated skull base approach and technique were required in the present case.

\section{Conflict of Interest}

The authors have nothing to disclose.

\section{References}

1 Caldarelli M, Massimi L, Kondageski C, et al. Intracranial midline dermoid and epidermoid cysts in children. J Neurosurg 2004; 100:473-480

2 Danaila L, Carp N. Dermoid tumour of the fourth ventricle with hyperdense aspect demonstrated on CT scan. Case report. Neurol Psychiatr (Bucur) 1989;27:231-236

3 Karabulut N, Oguzkurt L. Tetraventricular hydrocephalus due to ruptured intracranial dermoid cyst. Eur Radiol 2000; 10:1810-1811

4 Lunardi P, Missori P. Supratentorial dermoid cysts. J Neurosurg $1991 ; 75: 262-266$

5 Lunardi P, Missori P, Rizzo A, et al. Chemical meningitis in ruptured intracranial dermoid. Case report and review of the literature. Surg Neurol 1989;32:449-452

6 Oursin C, Wetzel SG, Lyrer P, et al. Ruptured intracranial dermoid cyst. J Neurosurg Sci 1999;43:217-220

7 Pant I, Joshi SC. Cerebellar intra-axial dermoid cyst: a case of unusual location. Childs Nerv Syst 2008;24:157-159

8 Uppal HS, D'Souza AR, De R, et al. Dermoid cyst of the infratemporal fossa. J Laryngol Otol 2002;116:150-152 
9 Ammirati M, Delgado M, Slone HW, et al. Extradural dermoid tumor of the petrous apex. Case report. J Neurosurg 2007; 107:426-429

10 Hong SW. Deep frontotemporal dermoid cyst presenting as a discharging sinus: a case report and review of literature. Br J Plast Surg 1998;51:255-257

11 Sichel JY, Dano I, Halperin D, et al. Dermoid cyst of the eustachian tube. Int J Pediatr Otorhinolaryngol 1999;48:77-81

12 Kocaeli H, Korfali E, Dogan S, et al. Sylvian cistern dermoid cyst presenting with dysgeusia. Acta Neurochir (Wien) 2009; 151:561-563

13 Terai T, Henmi T, Kanematsu Y, et al. Adult onset tethered cord syndrome associated with intradural dermoid cyst. A case report. Spinal Cord 2006;44:260-262

14 Ahuja R, Azar NF. Orbital dermoids in children. Semin Ophthalmol 2006;21:207-211

15 Fujimaki T, Miyazaki S, Fukushima T, et al. Dermoid cyst of the frontal bone away from the anterior fontanel. Childs Nerv Syst 1995;11:424-427
16 North KN, Antony JH, Johnston IH. Dermoid of cavernous sinus resulting in isolated oculomotor nerve palsy. Pediatr Neurol 1993; 9:221-223

17 Sturiale CL, Mangiola A, Pompucci A, et al. Interdural giant dermoid cyst of the petrous apex. J Clin Neurosci 2009; 16:1498-1502

18 Detweiler MB, David E, Arif S. Ruptured intracranial dermoid cyst presenting with neuropsychiatric symptoms: a case report. South Med J 2009;102:98-100

19 El-Bahy K, Kotb A, Galal A, et al. Ruptured intracranial dermoid cysts. Acta Neurochir (Wien) 2006;148:457-462

20 Warakaulle DR, Anslow P. Differential diagnosis of intracranial lesions with high signal on T1 or low signal on T2-weighted MRI. Clin Radiol 2003;58:922-933

21 Drolshagen LF, Standefer M. Dense dermoid cyst of the posterior fossa. AJNR Am J Neuroradiol 1991;12:317

22 Nakagawa K, Ohno K, Nojiri T, et al. Interdural dermoid cyst of the cavernous sinus presenting with oculomotor palsy: case report [in Japanese]. No Shinkei Geka 1997;25:847-851 